rev.relac.int.estrateg.segur.12(1):209-236,2017

\title{
INTERVENCIONISMO Y MEDIO AMBIENTE: EL CASO DE LA AMAZONÍA BRASILEÑA*
}

\author{
Gisela da Silva Guevara**
}

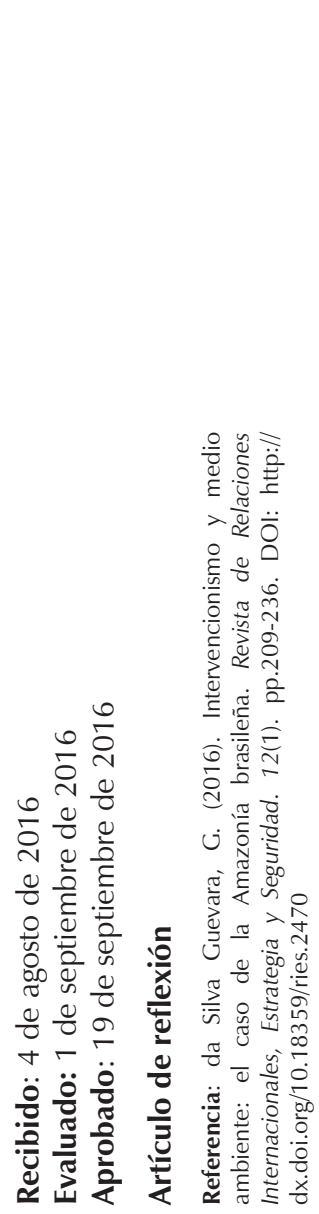

\section{Resumen}

El presente artículo busca debatir conceptos tales como la internacionalización de la Amazonía y la soberanía de los Estados en el ámbito ambiental, reflexionando sobre los aportes de autores que se dedicaron a estos temas. La metodología es cualitativa, utilizando diversidad de obras y artículos académicos, así como fuentes primarias, donde se puede verificar la variedad de posiciones sobre los mencionados conceptos. Reflexionaremos acerca del significado del concepto de internacionalización de la Amazonía, por una parte visto del Sur, sobre todo desde Brasil, y por otra, de los países del Norte, debatiendo las implicaciones de la confrontación de enfoques entre los países desarrollados y las naciones en vías de desarrollo para las relaciones internacionales. Partimos de la premisa de que el

\footnotetext{
Artículo elaborado en el marco del proyecto de investigación desarrollado en el Centro de Investigaciones y Proyectos Especiales (CIPE) de la Universidad Externado de Colombia.

** Docente-investigadora de la Facultad de Finanzas, Gobierno y Relaciones Internacionales de la Universidad Externado de Colombia. Magíster en Estudios de Seguridad e Inteligencia de la Universidad Brunel, Reino Unido y Doctora en Ciencia Política y Relaciones Internacionales de la Universidade do Minho, Portugal. gisela.silvaguevara@gmail.com.
} 
obstáculo para un consenso entre el Norte y el Sur sobre la internacionalización de la Amazonía brasileña se basa en nociones divergentes de soberanía y responsabilidad ambiental de los Estados, las cuales hasta nuestros días no pudieron ser resueltas.

Palabras clave: Consenso Norte-Sur; Internacionalización de la Amazonía; Recursos naturales; Soberanía del Estado

\title{
INTERVENTIONISM AND ENVIRONMENT: THE BRAZILIAN AMAZON CASE
}

\begin{abstract}
This article intends to debate on concepts such as the internationalization of the Amazonian region and the sovereignty of the States concerning the environmental sphere, reflecting on contributions from authors that have discussed these topics. The methodology used is a qualitative one, resorting to diverse books and academic articles as well as to primary sources through which the variety of positions on the aforementioned concepts can be seen. In here, there is a reflection on the meaning of the concept "Internationalization of the Amazon" as seen from, on the one hand, the South - especially Brazil -, and, on the other, the Northern countries, debating on the implications for international relations of the contrasting approaches between developed countries and developing nations. The starting point is the premise of the obstacle for a consensus between North and South regarding the internationalization of the Brazilian Amazonian region being based on divergent notions about sovereignty and social responsibility from the States, notions that, up to date, have not been leveled.
\end{abstract}

Keywords: North-South consensus; Internationalization of the Amazonian region; Natural resources; State sovereignty.

\section{INTERVENCIONISMO E MEIO AMBIENTE: O CASO DA AMAZÔNIA BRASILEIRA}

\section{Resumo}

O artigo presente procura conceitos tais como a internacionalização da Amazônia e a soberania dos Estados no âmbito ambiental, reflexionando sobre os aportes de autores que se dedicaram a estes temas. A metodologia é qualitativa utilizando diversidade de obras e artigos acadêmicos, assim como fontes primárias onde 
se pode verificar a variedade de posições sobre os conceitos mencionados. Reflexionaremos sobre o significado do conceito de internacionalização da Amazônia, por uma parte visto do Sul, sobre tudo desde o Brasil e por outra dos países do Norte, debatendo as implicações da confrontação de enfoques entre os países desenvolvidos e as nações em via de desenvolvimento para as relações internacionais. Partimos da premissa que o obstáculo para um consenso entre Norte e o Sul sobre a internacionalização da Amazônia brasileira se baseia nas noções divergentes de soberania e responsabilidade ambiental dos Estados, as quais até os nossos dias não puderam ser resolvidos.

Palavras chave: Consenso Norte-Sul; Internacionalização da Amazônia; Recursos naturais; Soberania do Estado

\section{Introducción}

La Amazonía brasileña ocupa más del $60 \%$ de territorio de Brasil, correspondiendo a unos 5,2 millones de $\mathrm{km}^{2}$. El país detiene $18 \%$ de las reservas de agua dulce del planeta, estando la mayor parte de estas en la Amazonía (Becker, 2004). El territorio pan-amazónico es compartido por ocho países, estando $68 \%$ de la cuenca amazónica y de las selvas tropicales en territorio de Brasil (Castro, 2008).

Elpresenteartículobuscadebatirconceptos tales como la internacionalización de la Amazonía y la soberanía de los Estados en el ámbito ambiental, reflexionando sobre los aportes de autores que se dedicaron a estos temas. La metodología es cualitativa, utilizando diversidad de obras y artículos académicos, así como fuentes primarias, donde se puede ver la variedad de posiciones sobre los mencionados conceptos.

Brasil se encuentra en el grupo de naciones megadiversas del mundo, grupo de casi veinte naciones, donde también se encuentra Colombia, las cuales se pautan por el mayor índice de biodiversidad ${ }^{1}$ mundial. No tan mencionado, pero de igual importancia, es la etnodiversidad, la cual, según la estimaciones de Costa Filho (2003) para la Pan-Amazonía podría alcanzar 453 lenguas nativas².

Una de las razones para el desconocimiento de los pueblos

Según el Convenio sobre la Diversidad Biológica (Naciones Unidas, 1992, pp. 3-4) se entiende "por 'diversidad biológica' la variabilidad de organismos vivos de cualquier fuente, incluidos, entre otras cosas, los ecosistemas terrestres y marítimos y otros ecosistemas acuáticos y los complejos ecológicos de los que forman parte; comprende la diversidad dentro de cada especie, entre las especies y los ecosistemas."

2 Triangulando con la información de la Organización de Cooperación Amazónica (OTCA), esta última menciona 420 pueblos indígenas que hablan 86 lenguas y 650 dialectos. 
indígenas se ata con la falta de investigaciones sobre estos debido al no dominio de lo escrito, pero también con razones históricas que llevaron los colonizadores de los espacios amazónicos a buscar ganancias con sus riquezas sin tener en cuenta estos a pueblos.

Según la geógrafa Bertha Becker (2004, p. 21), en la Amazonía se ha dado un "proceso de mercantilización de la naturaleza", imperando la percepción de que el espacio amazónico era "una frontera de recursos, esto es, área de expansión de poblamiento y de economías nacionales" ${ }^{\prime 3}$ que el Estado debería garantizar a toda costa para asegurar su propia soberanía. Ya en los años cuarenta del siglo pasado, bajo Getúlio Vargas, en el contexto de la Segunda Guerra Mundial, Brasil se había comprometido, en el marco de los Acuerdos de Washington ${ }^{4}$ a suministrar caucho a los aliados. Sin embargo, otro imperante, quizás de mayor relevancia para Vargas, tenía que ver con el slogan "Conquistar la tierra, dominar el agua, sojuzgar la selva" (cit. en Secreto, 2007, p. 129). Se trataba de presentar la Amazonía como un espacio que abriría nuevas posibilidades económicas y sociales a los que no las tenían (Secreto, 2007). Se iría a fomentar entonces la migración de trabajadores del empobrecido nordeste del país hacia la Amazonía. Para eso había que dominar la naturaleza amazónica y "ocupar los espacios vacíos de esta" (Secreto, 2007, p. 117).

Existía un componente adicional en Vargas y su aparato propagandístico en pro de la ocupación y dominación de espacios amazónicos. Se trataba de excluir a pueblos extranjeros que amenazaban eventualmente apropiarse de estos (Andrade, 2010). De hecho, en los años treinta y cuarenta del siglo XX, Getúlio Vargas impulsó una política que consolidase una zona de frontera amazónica, la faixa (franja) a la cual sería otorgado un status especial. En esta franja, la concesión de tierras estaba sujeta a autorizaciones especiales (Anatol, 2011). Ya entonces, el cuidado con aspectos de defensa y de seguridad de la frontera amazónica eran considerados prioritarios.

Bajo el régimen autoritario brasileño (1964-1985), el ejercicio de la soberanía pasa a estar estrechamente atado a una gran inversión del Estado, por la cual sean construidas en la región amazónica infraestructuras tales como carreteras. Para Conca (1995) pasa a ser una constante, desde mediados del sesenta, procesos pautados por la

Traducción propia.

Cuando EE. UU. entró en la Segunda Guerra Mundial, Brasil se comprometió con este país en proveer su industria bélica con minerales y caucho. El Gobierno brasileño incentivó entonces el reclutamiento de trabajadores para la Amazonía. 
expansión de la frontera o destrucción de la selva. Interactuando con estos procesos subyace el dilema de fortalecer el poder del Estado y a la vez no poder salir de la dependencia del país (Conca, 1995). Son fomentados entonces proyectos de gran magnitud para financiar en la Amazonía la agricultura, la ganadería a gran escala y la construcción de hidroeléctricas.

Conca (1995) recalca que la deforestación de la selva tropical conllevó paulatinamente a un debate sobre la problemática de la soberanía en las políticas ambientales de las naciones. En los ochenta y noventa se elevaron las voces de académicos, científicos y líderes políticos que comenzaron a cuestionar la responsabilidad ecológica de los Estados.

Con la Conferencia de las Naciones UnidasparaelMedioAmbiente,conocida como Conferencia de Estocolmo, en 1972, se llega a una declaración cuyo principio 21 generaría el debate relativo al principio de soberanía de los Estados sobre los recursos naturales propios, el cual establece que: "los Estados tienen el derecho soberano de explotar sus propios recursos en aplicación de su propia política ambiental y la obligación de asegurar que las actividades que se lleven a cabo dentro de su jurisdicción o bajo su control no perjudiquen al medio de otros Estados o de zonas situadas fuera de toda jurisdicción nacional. "(Declaración de Estocolmo sobre el Medio Ambiente Humano, 1972, Principio 21).

Para Priscilla de Passos (2009), la Conferencia de Estocolmo (1972) fue decisiva para impulsar en los países en desarrollo sus responsabilidades en hacerse cargo de asuntos ambientales. De hecho, dos años después, en Venezuela, Arnoldo Gabaldón proponía al presidente Carlos Pérez crear un Ministerio del Ambiente, modelo tomado en Colombia por el político Manuel Rodríguez ${ }^{5}$. Sin embargo, en Brasil la problemática ambiental desencadenaría suspicacias de que los países desarrollados anhelaban controlar las riquezas de la selva amazónica.

Los temores nutridos, entre otros, por círculos políticos y militares brasileños se ataban a presiones de los países desarrollados sobre los en vías en desarrollo en cuestiones ambientales. Para Shiguenoli Miyamoto (2008, pp. 74-75) Brasil era presentado en el palco internacional como "el gran villano" en lo concerniente a sus políticas supuestamente violadoras de la sostenibilidad ${ }^{6}$ de la Amazonía.

Información dada por Arnoldo Gabaldón y Manuel Rodríguez en una entrevista realizada el 1 de junio de 2016 a una cadena de televisión colombiana, Cable Noticias.

$6 \quad$ El concepto de desarrollo sostenible es definido en el informe Brundtland (Naciones Unidas, 1987) como "development that meets the needs of the present without compromising the ability of future generations to meet their own needs:" 
A finales de los años ochenta el presidente Sarney había refutado las críticas internacionales al país en cuanto a ser negligente en asuntos ambientales, afirmando que "Brasil nunca descuidó el problema ambiental y constantemente viene actualizando procedimientos y tomando iniciativas" (citado en Myamoto, 2008, p. 75). Adicionalmente a la problemática de las críticas contundentes que se hacían sentir contra el país en temas ambientales, se juntaba el temor de que la deuda externa ${ }^{7}$ del país conllevase a la cesión de parte de la Amazonía. De hecho, en 1983 Margaret Tatcher proponía en el G-7, la venta del territorio amazónico, a cambio de que se perdonase la deuda de naciones sudamericanas. A finales de los ochenta, el presidente François Mitterrand sugería la "soberanía relativa" de la Amazonía (Teixeira da Silva, 2008).

En los años noventa un grupo de países que incluían a Estados Unidos (EE. UU.), Francia y Holanda avanzaban con la propuesta del debt-for-nature, mediante la cual se perdonaría una parte de la deuda externa de Brasil en cambio de que el país aceptase proyectos internacionales con la finalidad de conservar la naturaleza. Adicionalmente el G-7 anunciaba un proyecto presupuestado en un poco más de 1,5 billones de dólares para financiar el desarrollo sostenible de la Amazonía (Conca, 1995). En esos años, como revela una revisión de la prensa brasileña, pasaría a ser recurrente los artículos sobre la eventual amenaza de la internacionalización de la Amazonía (Ryan, 1993).

A fin de contraponerse a ser tratado como un Estado en vías de desarrollo solamente recipiente de normas ambientales, las cuales, eventualmente, según la perspectiva de los tomadores de decisión brasileños, podría afectar su soberanía, Brasil decide pasar a la ofensiva y ser más activo en la Conferencia de las Naciones Unidas sobre Medio Ambiente y Desarrollo, la cual tendría lugar en 1992, en Rio de Janeiro. Veinte años antes, en Estocolmo ya había estado atento a los desarrollos de pautas ambientales. Pero esta vez, Brasil no estaba dispuesto a ser remitido a la posición de villano (Ricupero i.a., 1995).

El fantasma de la internacionalización de la Amazonía, el cual en la práctica se plasmaría en la pérdida de la soberanía absoluta de Brasil en ese espacio, se haría sentir fuertemente en medios militares, académicos y políticos brasileños, llegando a puertas del siglo XXI, cuando en la Estrategia Nacional de Defensa, publicada en

Entre 1983 y 1987 Brasil pagaba altos montos para el servicio de su deuda externa. Adicionalmente, el país tenía que realizar nuevos préstamos, los cuales llegaban por año a 10 billones de dólares (Ricupero, i.a., 1995). 
$2008^{8}$, se mencionaba "Quien cuida de la Amazonía brasileña, a servicio de la humanidad y de sí mismo, es Brasil." (Ministerio da Defesa, Brasil, 2008, p. 14).

El presente artículo busca analizar de qué forma las divergencias de enfoques relativos a la comprensión de espacios tales como la Amazonía brasileña han causado entre el Norte y el Sur conceptualizaciones diferentes y así mismo divergentes en cuanto a la forma como los Estados deben intervenir en asuntos ambientales. Consideramos que es necesario dar más atención a estos aspectos, pues no siempre la literatura especializada es clara al respecto.

En la primera parte del presente texto averiguaremos las distintas posiciones de militares, políticos y académicos brasileños sobre qué significa la Amazonía brasileña y su defensa para la soberanía del país, sobre todo frente a las supuestas amenazas de los países del Norte.

En la segunda parte analizaremos el significado y las implicaciones para la Amazonía brasileña del debate académico sobre las posibilidades de intervenir en un territorio soberano cuando esté en riesgo la seguridad ecológica.
En la tercera y última parte reflexionaremos sobre el significado del concepto de internacionalización de la Amazonía, por una parte visto del sur, sobre todo desde Brasil, y por otra, desde los países del Norte, debatiendo las implicaciones de la confrontación de enfoques entre los países desarrollados y las naciones en desarrollo, para las relaciones internacionales.

\section{Soberanía y visiones de defensa sobre la Amazonía brasileña}

En la Estrategia Nacional de Defensa (Ministerio da Defesa, Brasil, 2008, p. 14), se enfatiza en el punto 10 que Brasil busca dar prioridad a la Amazonía, rechazando "por la práctica de actos de desarrollo y de defensa, cualquier intento de tutela sobre sus decisiones al respecto de preservación, de desarrollo y de defensa de la Amazonía", recalcando que la nación no "permitirá que organizaciones o individuos sirvan de instrumentos para extranjeros políticos o económicos- que quieran debilitar la soberanía brasileña".

Los derechos y las responsabilidades de los Estados en aspectos ambientales han sido, desde por lo menos la segunda mitad del siglo XX hasta la

En 2012 la Estrategia Nacional de Defensa fue actualizada. 
actualidad, un punto de gran discordia entre los países desarrollados del Norte y los países en vías de desarrollo. En la década del setenta, en Brasil, los gobiernos militares nutrieron una "convicción estratégica" que sería fundamental ocupar la Amazonía, pues lo que estaba en causa era la defensa de la soberanía del país en ese espacio. Los decisores políticos crearon entonces un "imaginario de amenaza y codicia internacional" que tendría que ser frenada por una "movilización nacionalista" (Lima de Medeiros, 2012, pp. 134-135).

Bajo el slogan "ocupar para no entregar" se ideó a mediados de los años ochenta el Plan Calha Norte (PCN), el cual tenía en ese entonces el objetivo de fortalecer la presencia militar de Brasil en la parte norte de la Amazonía, con el fin de detener supuestas pretensiones de los países desarrollados en esos ricos territorios (Foresta, 1992). El PCN sigue vigente hasta nuestros días y actualmente el Ministerio de Defensa (2016) define su objetivo principal como "la manutención de la soberanía de la Amazonía, contribuyendo a la promoción de su desarrollo ordenado y sostenible".

En el 2006, según afirmaciones que Al Gore había proferido en el pasado, estas fueron reproducidas en periódicos norteamericanos, afirmando: "Contrario a lo que los brasileños piensan, la Amazonía no es su propiedad, sino pertenece a todos nosotros ${ }^{\prime \prime}$, los círculos políticos brasileños reaccionaron de forma vehemente. Marina Silva, la enérgica ministra del Medio Ambiente de Lula da Silva afirmaba: "La Amazonía no está a la venta" (Folha de São Paulo, 17/10/2006/). En ese mismo año David Miliband, entonces ministro del Medio Ambiente de Tony Blair había expresado sus dudas sobre la capacidad de Brasil en proteger la Amazonía. A finales de ese mismo año, Miliband había sugerido la creación de un trust internacional que tendría la función de manejar la selva amazónica (The Guardian, 19/10/2006). Como respuesta a este tipo de sugerencias por parte de políticos de gobiernos del Norte, Lula da Silva había afirmado: "La Amazonía es nuestra" (Estadão, 06/07/2007).

Más recientemente, un militar de alto rango del ejército brasileño, el general de brigada Luís Eduardo Rocha Paiva, expresaba la convicción de que Brasil debería estar muy atento a la posibilidad de que se produjeran en la Amazonía conflictos con actores extranjeros debido, entre otros, a la riqueza de sus recursos. Asimismo, Rocha Paiva alertaba sobre la posibilidad de injerencia de potencias extra-regionales

Afirmaciones proferidas originalmente en 1989, reproducidas, de nuevo, en el New York Times, 18 de mayo de 2005, cuando la polémica de posibles ambiciones de EE. UU. en la Amazonía se reavivó. 
en este espacio (Secretaría de Asuntos Estratégicos, 2012).

Duarte enfatiza entre los motivos fundamentales para Brasil centrarse en la dimensión militar el que "deriva en parte de la preocupación con la soberanía y la integridad del territorio brasileño, muy particularmente con el inmenso patrimonio que representa para Brasil las amazonias verde a azul ${ }^{10}$, tanto observadas como codiciadas por poderosas potencias externas" (2014, p. 65).

Según el académico Shigenoli Miymoto (2008), ha sido la combinación de varios factores los que llevaron a Brasil a centrar mayor atención en la frontera amazónica. Entre estos estaba el hecho de que Brasil y Argentina dejaron de percibirse mutuamente como amenazas, lo cual conllevó a que la frontera platina, al sur, permitiese un alivio en la presión militar-estratégica. El segundo aspecto pasó a ser un mayor cuidado con la selva tropical y las críticas dirigidas al país por la degradación ambiental. Adicionalmente, en la pos Guerra Fría, el Plan Colombia, firmado en el 2000 para proveer a Colombia de recursos en el combate del narcotráfico, llevaría a Brasil a percibir dicha ayuda como una puerta abierta a una injerencia en la Amazonía brasileña (Da Silva Guevara, 2011).
Hay por parte de algunos académicos brasileños escepticismo en cuanto a la asistencia o cooperación internacional con Brasil en asuntos ambientales. En una tesis de posgrado, da Silva (2012, p. 166) afirma que "por mucho que se construyan mecanismos cooperativos, de acción conjunta internacional, los procesos de negociación de [...] medidas ecológicas son pautados no [...] por la solidaridad y conciencia ambiental, sino por el cálculo económico, por la relación de fuerzas e influencias de poder".

Con Lula da Silva (2003-2010), el Plan Calha Norte amplió su área de actuación, que pasó de 7413 km de frontera a $10938 \mathrm{~km}$, o sea $25 \%$ del espacio nacional (Nascimento, 2008). Se trata de un área de fundamental importancia geoestratégica para Brasil, pues colinda con vecinos como Colombia, Venezuela, Perú y Bolivia, entre otros. Pero adicionalmente, según el general Rocha Paiva, tratándose la Amazonía de una región no completamente integrada en el espacio nacional, y siendo la soberanía carioca cuestionada internacionalmente, la injerencia de potencias extra-regionales, carentes de recursos, podría adicionar un factor de inestabilidad para la soberanía de la nación. A todo esto el general agrega que la intención de EE. UU. en Sudamérica de no ceder espacio a

10 La Amazonía azul se refiere al Atlántico sur bajo soberanía de Brasil, el cual comprende 4500000 km2, o sea, un 52\% del espacio continental brasileño. 
rivales, como China, podría potenciar más conflictos en la región (Secretaría de Asuntos Estratégicos, 2012).

Para el general Eduardo Dias Villas Boas (2013) hay que cambiar el enfoque hacia la selva. Se trata de verla como origen de bienestar y no de obstáculo al desarrollo. El rol de la Amazonía para Brasil es según Villas Boas, entre otros, el de contribuir a su vocación de liderazgo en la región, alimentar el proyecto integracionista y cooperar a dar soluciones a las problemáticas del medio ambiente y cambio climático. Sin embargo, para el autor sigue todavía vigente el ambiente de distanciamiento entre los vecinos amazónicos, prolongándose la situación de abandono de las fronteras. Faltaría así para la región una visión común de lucha contra los ilícitos y respeto al trato de las comunidades indígenas (Villas Boas, 2013).

En 2013, en el Gobierno de Dilma Rousseff, fueron escuchadas las voces de altos rangos militares sobre la situación de la débil presencia del Estado en la Amazonía. La presidenta autorizó en ese año la creación del Comando Militar del Norte (CMN) con el fin de proteger, entre otros, infraestructuras estratégicas o combatir la explotación ilegal de recursos naturales. El nuevo CMN, ubicado en la Amazonía oriental, pasaba a ser el octavo comando del Ejército, con base en la ciudad de Belem. Entre el 30 de mayo y el 10 de junio de 2016, el CMN realizó la Operación
Cabo Orange, en colaboración con la Guyana francesa, con el fin de combatir ilícitos fronterizos y crímenes ambientales (Ejército brasileño, 2016). El Comando también ampara a la población con acciones cívicosociales, como apoyo médico. Sin embargo, siguen los temores por parte la brasileña que la "cuestión ambiental es el tema que permanece como una de las preocupaciones de la humanidad y que países detentores de gran diversidad [...]", como Brasil, "pueden volverse objeto de interés internacional" (Secretaría de Asuntos estratégicos, 2013, p. 13).

Uno de los aspectos que sigue despertando las suspicacias de Brasil cuanto a que su soberanía en la Amazonía puede estar en riesgo tiene que ver con lo que Ayoob (2002) enfatizó ser la problemática de la asimetría en las relaciones internacionales. Este autor relevó que las diferencias de hard power (poder duro) y soft power (poder blando) entre las grandes potencias y las naciones más débiles en el sistema internacional plasman en las relaciones entre países las discrepancias jerárquicas del sistema mundial. Entre ellas está la diferencia de capacidades materiales y de conocimiento. Esto implica, entre otros, que normas internacionales definen internamente las trayectorias de dichos Estados subalternos.

En Brasil, las mencionadas discrepancias de poder en el sistema internacional fueron, quizás más que en cualquier 
otra nación sudamericana, tomadas muy en serio por parte de militares, políticos y académicos. Por ejemplo, para el académico Meira Mattos (1995) las naciones más poderosas ampliaron sus intereses y sus estrategias de seguridad en el ámbito externo, traspasando fronteras nacionales, las cuales contribuyen a fortalecer su posición en el sistema internacional. Como resultado de esta disparidad de poder en el sistema mundial, según el mismo autor, es relevante la asimetría entre el Norte y el Sur, socavando la soberanía de Brasil en la Amazonía.

El temor por parte de las elites brasileñas de que la Amazonía llegase, en el futuro, a salir de la órbita soberana del país conllevó notablemente a Brasilia a tomar iniciativas contundentes de monitoreo y vigilancia de ese espacio. Con el Plan Calha Norte, ya anteriormente mencionado, y la implementación del SIVAM-SIPAM (Sistema de Vigilancia de la Amazonía - Sistema de protección de la Amazonía), se buscaba (y busca) controlar la Amazonía. EI SIVAM tiene el objetivo de, con apoyo en radares, colectar información sobre el espacio amazónico que va desde la deforestación hasta movimientos ilícitos aéreos, expansión de espacios agropecuarios y ubicación y desplazamiento de pueblos indígenas. Para el investigador Durbens Nascimento (2010, p. 184), ambos, Plan Calha Norte y SIVAM, están estrechamente articulados, con la finalidad de "defender, proteger y garantizar la integridad del territorio nacional $[\ldots] " .$.

Para Duarte la forma como Brasil visualiza la realidad internacional es no solamente "como correcta o incorrecta, sino como un espacio peligrosamente hostil movido por fuerzas alienígenas, que condicionan desfavorablemente el comportamiento de la mayoría de los actores y que deben, en consecuencia, ser afrontados" (2014, p. 68).

Vimos así en esta primera sección del texto que la Amazonía brasileña tiene no solamente una importancia fundamental para la humanidad, sino para el mismo Brasil. Sin embargo, la riqueza y el alto índice de biodiversidad de este espacio no siempre fueron preservados de forma tan cauta como sería deseable. Por otro lado, Brasil acusa a las potencias desarrolladas del Norte de nutrir intereses respecto a la Amazonía que podrían, eventualmente, socavar su soberanía. En la próxima sección averiguaremos cuestiones cruciales concernientes al debate sobre las posibilidades de intervención externa en cuanto a aspectos ambientales en la Amazonía.

Intervenir o no intervenir: aspectos conceptuales sobre el medio ambiente A mediados del 2015, un artículo de Richard Schiffman (2015), experto en asuntos ambientales, mencionaba los retrocesos que se habían concretado en el combate a la tala de árboles de las selvas tropicales, en el período 
transcurrido entre 1990 y 2010. En este mismo espacio temporal, la tala había aumentado en un $62 \%$ en el mundo. En cambio, en Brasil estos índices habían bajado entre 2004 y 2011. Según el autor, esto se debía a la implementación de normas ambientales más estrictas y a medidas que buscaban controlar el cultivo de soja en zonas deforestadas.

No obstante lo mencionado anteriormente, a partir de la segunda mitad del 2014, la tala de árboles se duplicó en la Amazonía brasileña, comparada con el mismo periodo del año anterior (Schifmann, 2015). Por otro lado, datos oficiales (Ministério do Meio Ambiente, 2016) indican que a partir del 2004 el Gobierno federal, creando el Plan de Acción para la Prevención y Controle de la Deforestación de la Amazonia Legal (PPCDAM), había beneficiado a 25 millones de habitantes y reducido en el 2012, un 84 \% la deforestación, comparado con el 2004.

Si bien es cierto que algunos progresos y esfuerzos notables se pueden registrar en la Amazonía brasileña en cuanto al combate a la deforestación, lo cierto es que investigadores del Instituto de Investigación Ambiental de la Amazonia (IPAM en sus siglas en portugués) alertan para la necesidad de Brasil reducir sustancialmente su deforestación (IPAM, abril de 2016). Para lo que nos ocupa en el enfoque del presente texto están, entre otros, las diferencias de posiciones frente a problemáticas ambientales de las selvas mundiales entre los países desarrollados y las naciones en desarrollo, las cuales pasaremos a analizar a continuación.

Para Conca (1995) existen diferencias fundamentales de posición entre el Norte y el Sur cuanto a las problemáticas antes mencionadas. Entre estas está la argumentación defendida por países desarrollados según la cual, teniendo en cuenta que las selvas son "bienes globales" (global commons), asegurando así un bien común de la humanidad, existen, por consiguiente implicaciones que podrían justificar, en algunos casos extremos, la intervención de la comunidad internacional. En cuanto a estas propuestas los países del Sur reaccionan negativamente haciendo contrapropuestas que, a su vez, son mal recibidas por el Norte. Así pasó, por ejemplo, cuando gobiernos de espacios selváticos tropicales propusieron que las negociaciones generales sobre selvas debían incluir también zonas temperadas (Conca, 1995); una posición que fue rechazada por las Naciones Unidas, pero que en la realidad se basa en el principio de la reciprocidad.

La "Responsabilidad de Proteger" (R2P, en inglés), la cual fue plasmada en el Informe de la Comisión Internacional sobre la Intervención y la Soberanía Estatal (ICISS, 2001), tiene como una de sus premisas básicas que "La soberanía estatal implica responsabilidad y su principal responsabilidad para la 
protección de su pueblo radica en el mismo Estado" (ICISS, 2001, p. XI). La R2P debería justificar intervenciones militares, en serios casos de violaciones de derechos humanos o genocidio, pero ha suscitado acalorados debates. Más recientemente llevó a los académicos a cuestionar si el principio no debería ser también aplicado a asuntos ambientales.

Stec (2010) coloca la posibilidad de que la R2P podría aplicar también a temáticas comunes ambientales. En este caso se abriría la posibilidad a que, cuando el Estado fracasa en sus responsabilidades de proteger la herencia común natural, otros estados u organizaciones puedan intervenir. Así, la "preocupación común", common concern, podría estar dando paso a una transición por la cual Estados con soberanía casi absoluta podrían estar sujetos a restricciones a dicha soberanía.

Para Stec (2010, p. 368) es fundamental la transición del "interés común de Estados" para "la preocupación común de la Humanidad", lo cual "implica valores que están por encima y más allá del interés de los 'Estados'”. Por su vez Robyn Eckersley (2009) considera que la "intervención ecológica" puede ser un corolario de la "defensa ecológica". La autora define como intervención ecológica "la amenaza o uso de la fuerza como respuesta a la amenaza a un daño serio ambiental, incursionando en el territorio de un Estado víctima." (2009, p. 131).

Mientras en universidades del Norte se desarrollaba la conceptualización sobre las modalidades de intervenir por parte de la comunidad internacional en ámbitos ambientales, el Sur Global ${ }^{11}$, centrado en América del Sur, liderado por Brasil, publicaba en el 2006 los resultados del grupo de trabajo de Tarapoto, por el cual se llegaba a 15 indicadores priorizados relativos a la sostenibilidad de la selva amazónica. El llamado proceso de Tarapoto reclamaba tener un marco regional, el de la Organización del Tratado de Cooperación Amazónica (Ministério de Meio Ambiente, 2006). El mensaje político parecía ser el de que el Sur también estaba capacitado para producir trabajo científico y parámetros que permitiesen proteger las selvas tropicales. Aunque también es destacable que, a nivel internacional, la FAO (en español, Organización de las Naciones Unidas para la Alimentación y la Agricultura) apoyaba desde 1995 el proceso de

11 El Sur Global se refiere originalmente a la división entre el Norte y el Sur en términos políticos y económicos, herencia de la Guerra Fría, que había dividido el mundo entre el Norte, países desarrollados y el Sur, países del llamado Tercer Mundo. A finales del siglo XX se generó un movimiento de cooperación Sur-Sur, el cual tenía características no solamente económicas, sino que también buscaba disminuir la asimetría entre Norte y Sur. Altinbas (2013, p. 46) menciona que "Efforts to create a multipolar world are significant in the sense that they foreshadow meaningful relational changes vis-à-vis the North-South." 
formulación de criterios e indicadores de gestión sostenible para la selva amazónica (García, 2011).

El contexto para la producción de trabajo en pro de las bases científicas de la sostenibilidad de la selva tropical era propicio a inicios del siglo XXI. En el 2002 tenía lugar la Cumbre Mundial sobre el Desarrollo Sostenible, en Johannesburgo, Sudáfrica, y pasados dos años se daba la cuarta reunión del Foro de las Naciones Unidas sobre los Bosques, en Ginebra. Pero mientras nobles intenciones venían a la luz en términos ambientales, tanto a nivel mundial como regional se levantaban críticas sobre la falta de efectividad de la cooperación en términos ambientales. Por un lado, entre el Norte y el Sur, y por otro entre las naciones del mismo Sur.

Eduardo Villas Boas (2013, p. 52) recalca que la riqueza de la Amazonía puede también llevar "la opinión pública internacional, ávida por cuestionar nuestra capacidad de gestionar lo que ya es considerado como patrimonio de la Humanidad". Villas Boas (2013, p. 58) parece también demostrar escepticismo cuanto a las organizaciones regionales centradas en la Amazonía, afirmando: "Se constata que tanto la OTCA como la IIRSA" tampoco "disponen de recursos capaces de gestionar efectos concretos".

Parece así mantenerse el dilema entre la importancia acordada a los bosques amazónicos y la dificultad de cooperar mundial y regionalmente al respecto. En documento de la Organización de las Naciones Unidas para la Agricultura y Alimentación (FAO, 2004, p. 52) se hace un llamado por el cual "los bosques amazónicos son génesis y futuro de las poblaciones de la región. Son hábitat vital y proveedores de sustento y contienen culturas milenarias y conocimientos ancestrales."

Para Mische (1989) se hace necesario establecer parámetros de gobernanza relativos a la seguridad global ecológica. Para la autora, entre el ámbito de la gobernanza y el de la soberanía debe haber ámbitos de "responsabilidad compartida", con la finalidad última de que la humanidad pueda tener (todavía) un futuro. La autora argumenta que si bien es cierto que los Estados están en su buen derecho de exigir la explotación de los recursos naturales que se ubican en su territorio de jurisdicción, no pueden poner en riesgo "el funcionamiento de los sistemas naturales, mismo cuando están relacionados con actividades desarrolladas en su territorio." (1989, p. 219).

Lo anterior nos lleva a considerar la problemática de la Amazonía, en especial, en este texto, de la brasileña en aspectos que se atan a las responsabilidades que puedan compartir los Estados. Al respecto, García (2011) defiende que la comunidad de Estados tiene la "responsabilidad compartida" de proteger las selvas tropicales. Para 
la autora, "similares dificultades" en la cuenca amazónica conllevan a "intereses comunes". García plantea como modelo la estrategia de protección medioambiental en la Antártica, que fue pactada en 1991 por ocho naciones. La premisa de dicha cooperación había sido/ es que se trataba de una región muy particular, que ameritaba un enfoque especial. Desde 1989 se había acordado debatir "la implementación de medidas globales para la protección del medioambiente en la Antártica" (Rothwell, 1990, p. 284).

Aunque ha habido tentativas para comparar el caso de la Amazonía al de la Antártica es importante recalcar que, desde la perspectiva de Brasil, este territorio está bajo su única soberanía. Como lo recalca Herrera (2011, p. 19) “En el caso de la región amazónica es necesario tener en cuenta una diferencia vital: la región amazónica siempre ha sido parte del territorio brasileño".

El escollo de la cuestión es que los países en vías de desarrollo perciben varias formas de cooperación con actores extra-regionales en los territorios selváticos tropicales como una intervención o umedio de socavar su soberanía. En un informe del Consejo Económico y Social del Foro de las Naciones Unidas sobre los Bosques (Consejo Económico, Naciones Unidas, 2009) se estima que el $80 \%$ de la diversidad biológica en la Tierra está en los bosques, centrada sobre todo en los trópicos. Sin embargo, como recalca el mismo informe, "Para muchos países en desarrollo las actividades relacionadas con la ordenación sostenible de los bosques para su conservación y las zonas forestales protegidas tienen más probabilidades de éxito si esas actividades se recogen en los planes nacionales de desarrollo [...]" (Consejo Económico y Social, 2009, p. 2).

El economista brasileño Alfredo Costa-Filho (2003) releva que la conceptualización de que la Amazonía impacta los "servicios prestados por los ecosistemas", tales como la manutención de la biodiversidad y de la diversidad genética, la cual ha dado un significado especialmente estratégico a esta en el plano internacional. El autor (2003, p. 389) recalca igualmente que la evolución conceptual de "Medio Ambiente" ha sido fundamental en la importancia que la Amazonía ha tomado en los debates internacionales. Esto debido a que se trasladó el medio ambiente de "la condición de res nullius (no pertenece a nadie) para la de res omnium (que pertenece a toda la sociedad)" (p. 389). La problemática al respecto radica en que como anteriormente se mencionó, cuando los países en vías de desarrollo piden condiciones en igualdad de circunstancias, como el caso de aplicar los mismos parámetros, en igualdad de circunstancias, para la selva tropical a bosques temperados, el Norte rechaza la posibilidad. 
La temática antes mencionada va más allá de la simple reciprocidad. Como recalca el académico Adherbal Meira Mattos (2008, p. 134), "El principio de reciprocidad involucra una situación en la cual un Estado permite a otro un tratamiento igual o similar al que ese Estado le garantiza o promete. Hoy la tendencia es que los países subdesarrollados reivindican un tratamiento más favorable al de la simple reciprocidad, porque son subdesarrollados y necesitan, por consiguiente, de ventajas adicionales, que los países desarrollados no necesitan."

\section{La internacionalización de la Amazonía brasileña}

En el 2000, durante una ponencia realizada por el senador Cristovam Buarque en la Universidad de Nova Iorque, cuando fue cuestionado sobre lo que pensaba acerca de la internacionalización de la Amazonía, este contestó: "[...] como brasileño yo sería simplemente contra la internacionalización de la región amazónica. Mismo teniendo en cuenta que nuestros gobiernos no le dieron la importancia que este tesoro merece. Como humanista, entendiendo el riesgo que amenaza la destrucción de la Amazonía, podría considerar la internacionalización, tal como para el resto de lo que es importante para la humanidad"12 (Worldpress, 7/05/2008).

En el 2004, una propuesta realizada por Francia en un seminario sobre Gobernanza Mundial y Medio Ambiente relativo la creación de una Organización Mundial para el Medio Ambiente era rechazada por Brasil, por considerarla una tentativa de los países desarrollados para avanzar sus intereses económicos y ambientales en regiones con ricos recursos (Abdala, 2007). Un año después, tenía lugar una audiencia pública en el Senado brasileño, titulada "La internacionalización de la Amazonía: Riesgo real o temor sin fundamento". Como resultado de este debate y por temores expresados por parte de altos rangos militares al respecto de dicha internacionalización, el Gobierno federal suspendía dos años después la implementación de un proyecto para la conservación de la cuenca amazónica financiada por la Agencia Norteamericana para el Desarrollo Internacional (USAID, por sus siglas en inglés) (Rabinovici, 2008).

Más recientemente, en el 2015, el general Villas Boas, al ser invitado por el Senado para expresar sus posiciones sobre temas de la soberanía de Brasil en la Amazonía

12 Traducción del inglés, pues no fue posible hallar el original en portugués publicado en O Globo, 23 de octubre de 2000 . 
ante la Comisión de Relaciones Exteriores y de Defensa Nacional, criticó una propuesta presentada por una organización no gubernamental, para crear un corredor internacional "intocable" de recursos desde los Andes hasta el océano Atlántico, incluyendo la Amazonía brasileña. El general proponía más bien que se debía conciliar la preservación y el uso racional de las riquezas naturales. Contra la propuesta del corredor amazónico internacional argumentaba: "Este proceso (radicalismo por la preservación) es como combatir fantasmas, porque no sabemos de dónde vienen, quiénes son, qué hacen y cuáles son sus reales motivos" (Agência do Senado, 2015).

Los temores expresados por el general Villas Boas se atan con preocupaciones recurrentes en algunos medios militares brasileños en cuanto a la noción que defiende que la Amazonía debe ser considerada como "patrimonio de la humanidad", a la cual subyace la idea de que "un país, aunque soberano, no debe disponer unilateralmente sobre el futuro ecológico y ambiental de la humanidad" (Ovando, 27/06/2009). Por su parte, esta noción tiene que ver con el concepto de Bien Común de la Humanidad, el cual se basa en el principio de que, partiendo de la premisa de que ningún Estado debería apropiarse de recursos comunes, pues estos pertenecen a la humanidad, entonces estos recursos deberían ser gerenciados a nivel internacional (Abdala, 2007).
Países como Brasil se han opuesto en algunas ocasiones a la definición de un bien colectivo, como sería el caso de las selvas tropicales, pues temen que eso conlleve a la pérdida de soberanía sobre esos territorios. Como revela la académica Rabinovici (2008, p. 102) "La Amazonía en el imaginario de los brasileños es una inmensa reserva de recursos naturales, garantía para el futuro, vacío demográfico, vulnerable a las ambiciones de potencias internacionales". Abdala (2007) recalca que hay que considerar también el aspecto político del concepto de Bien Común Mundial, a fin de reevaluar el sentido de la cooperación internacional o de aspectos políticos transnacionales. Esto lleva entonces a que "No hay consenso sobre el concepto y la práctica de conservación de los patrimonios de la humanidad o de la soberanía compartida sobre recursos naturales considerados comunes" (Abdala, p. 86).

Ya en la Conferencia de las Naciones Unidas sobre Medio Ambiente y Desarrollo, que había tenido lugar en la ciudad de Rio de Janeiro, en 1992, se había permitido un paso más adelante en conceptos como el de desarrollo sostenible; por otro lado, dejado claras las divergencias de los intereses y los enfoques entre el Sur y el Norte. En el marco de la Conferencia se firmaría la Convención sobre Diversidad Biológica (CDB), la cual estipularía tres objetivos claros, a saber: 1) La conservación de la diversidad biológica; 2) La utilización 
sostenible de sus componentes; 3) La participación justa y equitativa en los beneficios que se deriven de la utilización de los recursos genéticos (Naciones Unidas, Convención sobre la Diversidad Biológica). Para llegar a un borrador final satisfactorio para todos los países, las negociaciones fueron largas. Del lado de los países en vías de desarrollo, el liderazgo de Brasil había sido decisivo. Uno de los escollos entre los países del Norte y los del Sur era el debate sobre si los recursos biológicos y genéticos deberían ser incluidos en los global commons (Correa do Lago, 2006).

Una de las problemáticas que se relacionan con lo anteriormente mencionado es la limitación de la eficacia del Estado - Nación en lidiar con temas globales como bienes comunes, gobernanza global o transnacionalismo (Abdala, 2007). Otra de las problemáticas es la de que si bien es cierto que los países en vías de desarrollo reconocen sus responsabilidades en contribuir al desarrollo sostenible, por otro lado priorizan la soberanía nacional, cuando se trata de resolver problemáticas ambientales. Sin embargo, no hay que ignorar como lo enfatiza Liftin (1997) que la cooperación en términos medioambientales alteró las premisas de la soberanía. La autora afirma al respecto que el principio de la no intervención parecería frenar los esfuerzos para proteger el medioambiente, sin embargo soluciones a problemáticas medioambientales contribuyen a su vez a la reconfiguración de la soberanía. Para la autora (1997, p. 171) "soberanía debería ser entendida como involucrando derechos, capacidades y responsabilidades [...]". $\mathrm{Si}$ bien es cierto que se reconoce las limitaciones de los Estados para lidiar con problemas ambientales, no podemos olvidar que Rio 1992 ha dado voz a las suspicacias de los países en vías en desarrollo, plasmando que "las medidas internacionales relativas a problemas ambientales transfronterizos o globales deben, en la medida de lo posible, basarse en el consenso internacional" (Declaración Rio 1992, principio 12). Por otro lado, algunos académicos como Mische (1989) consideran que, aunque parezca paradojal, temáticas relativas a seguridad ecológica global podrían incluso fortalecer la soberanía nacional.

La internacionalización de políticas forestales puede, según Scheurs (1997), dar una herramienta a los países en desarrollo para negociar con mayor margen de maniobra en el escenario internacional y fortalecer el principio de la soberanía. Así, todo depende de cómo los países del Sur global manejen los asuntos ambientales internacionalmente y, según consideramos, puedan cooperar para afirmar su voz en foros internacionales. Sin embargo, el debate sobre la internacionalización de las selvas, sobre todo tropicales, "está forzando los países en desarrollo 
a dar mayor atención a la preservación ambiental" (Scheurs, 1997, p. 185).

Krasner (1999) llama por su vez la atención para el hecho que no se puede ignorar que los Estados son profundamente asimétricos en poder, por lo que debería haber una prohibición básica de intervención. Quedaría la posibilidad de la "intervención por invitación". Sin embargo, esta opción podría conllevar a que, sin que los gobernantes que emiten la invitación tengan conciencia de eso, sean afectados los arreglos institucionales domésticos. Para otros autores como Bosselmann (2016) la cuestión fundamental de la perspectiva de la sostenibilidad es cómo el Estado moderno reacciona a retos globales medioambientales y cómo, a su vez, estos desafíos afectan a sus funciones básicas y deberes.

Bosselmann (2016), recalca que, partiendo de la premisa de que la variedad de principios medioambientales que comprenden desde el deber de cooperar hasta el desarrollo sostenible es demasiado débil, eso llevaría a la opción de que, tal como Kofi Amman había ya propuesto en los años noventa, se (re) conformase un Concejo de fideicomiso de las Naciones Unidas, por el cual los Estados miembros puedan ejercer "su fideicomiso colectivo en pro de la integridad del medioambiente global y de áreas comunes tales como los océanos, la atmósfera y el espacio" (citado en Bosselmann, 2016, p. 172). Lo que Bosselmann defiende es una restricción efectiva, por medio de un fideicomiso de Estados, en cuanto a la soberanía permanente sobre los recursos, la cual ya está plasmada en la Convención del Patrimonio Mundial. En este caso, tal como la Antártica, las selvas tropicales estarían bajo un fideicomiso de varias naciones. Bosselmann dice que la solución para proteger recursos de preocupación común (common concern) sería una reconfiguración de la soberanía territorial a través de la incorporación de derechos de fideicomiso. Esta idea, sin embargo, no sería del agrado de Estados como Brasil.

Kiss y Shelton (2007) mencionan que la protección del medioambiente se volvió una preocupación común (common concern) en el ámbito internacional a mediados del siglo XX. Posteriormente, "Al designar un asunto de preocupación común, esto desplazó el tópico de la jurisdicción exclusiva doméstica de los Estados y conllevó a legitimarlo como del ámbito de la jurisdicción internacional" (p. 124). Asimismo, en casos de referencia tales como el Tratado de la Antártica y del régimen antártico ${ }^{13}$ se evolucionó para aspectos más complejos y específicos de la protección ambiental

\footnotetext{
$13 \quad$ A partir de 1991.
} 
y del interés común (common interest) (Stec, 2010). Adicionalmente se desarrolló la argumentación de que recursos compartidos como el agua o ciertas especies son del ámbito del res communis (Bosselmann, 2016; Stec, 2010). Lo cierto es que como lo enfatizan Cárdenas y Cadena (2009) se desarrollaron nuevo enfoques a partir de conceptos tales como preocupaciones comunes, intereses comunes o patrimonio común de la humanidad. ¿Podría entonces la Amazonía brasileña ser justificada como un tema de common concern y así ser internacionalizada?

Es innegable que la Amazonía y, en el caso particular al cual dedicamos este texto, la brasileña, fue y es afectada por procesos como la contaminación de las aguas, minería ilegal, deforestación y amenazas tales como el tráfico de drogas y otros ilícitos. Ya en un artículo publicado a finales de los años setenta, el peruano Mercado Jarrín (1978, p. 18) había alertado que "El "tiempo" amazónico ha llegado, y si lo dejamos pasar, habremos cometido un pecado de abstención que las generaciones futuras nos censurarán con justicia".

Conca (1995) menciona que normas mundiales para el medioambiente pueden socavar la autoridad de un Estado. Sin embargo, si la reglamentación ambiental queda plasmada y codificada formalmente como de la responsabilidad del Estado, la legitimidad y el control de los Estados puede ser fortalecida.
El escollo del problema, como enfatiza el mismo autor, es que la temática de la protección ambiental es tan amplia y poco clara, que origina varias interpretaciones (y malinterpretaciones). Adicionalmente, agregaríamos que es indudable que las riquezas de la Amazonía conllevan a que la problemática de la asimetría de poder entre los Estados en el sistema internacional provoque suspicacias por parte de los países en vías de desarrollo.

Schrijver (2009, p. 73), a su vez, precisa que "la soberanía relativa y funcional siempre está reemplazando la soberanía absoluta", añadiendo que "sería una malinterpretación pensar que la soberanía del Estado siempre está obstruyendo la buena política para el medio ambiente y el desarrollo." Stec (2010) alerta, por su lado, que en algunos casos-estudio, como el de la internacionalización de la Antártica, si no se hubiese desarrollado conceptos como el de patrimonio común, tendríamos como mero resultado una explotación conjunta económica, sin preservar la naturaleza.

Solo muy recientemente los países en vías de desarrollo adquirieron conciencia ecológica sobre externalidades relativas a fenómenos transnacionales ambientales, tales como cambio climático, deforestación o pérdida de biodiversidad (Raghbendra y Whaley, 2007). Sin embargo, el debate sobre el binomio cooperación-recursos naturales viene 
siendo más presente en el plano internacional desde por lo menos la segunda mitad del siglo XX. Así, en 1959, en la Asamblea General de las Naciones Unidas, Filipinas enfatizó que la cooperación internacional debía ser voluntaria y no una imposición (citado en Schrijver, 2008, p. 61). Este tema en la realidad no fue resuelto desde entonces.

Desde los años noventa fueron tomando peso en América Latina los movimientos ambientalistas, muchos a favor de los indígenas. Sin embargo, en Brasil sigue a principios del siglo XXI la disputa entre "conceptos nacionalistas y propuestas de preservación ambientalista [...]" (Lima de Medeiros, 2012). La temática de la internacionalización de la Amazonía continúa vigente en el país y el viejo slogan "ocupar para no entregar" sigue teniendo gran importancia. La problemática es, sin embargo, iqué tipo de ocupación queremos para la Amazonía?

En el siglo XXI, en el marco del mandato de Lula da Silva, se lanzó el Plan Amazonía Sostenible (PAS), el cual tenía como objetivo principal un desarrollo según un nuevo modelo económico. Con el PAS se buscaba realizar "esfuerzos sistemáticos para internalizar [...] lineamentos estratégicos en el ámbito de políticas sectoriales" (Ministerio del Medio Ambiente Brasil, 2008, p. 91), donde fuese más estrecho el interactuar y la coordinación entre las esferas federal, de los Estados y la municipal. Asimismo, se buscaba reactivar la Superintendencia de Desarrollo de la Amazonía (SUDAM), la cual había sido creada en los años sesenta. Quizás el PAS tenía también la gran meta de "callar" críticas internacionales a Brasil. El SUDAM, de esta vez, se revivía bajo nuevos enfoques y competencias. Sin embargo, críticos del PAS como Madeira (2014) mencionan que las construcción de carreteras, puertos o hidroeléctricas en el espacio amazónico siguen, en la realidad, viejos modelos, los cuales no posibilitan a las poblaciones amazónicas un verdadero beneficio.

Es innegable que Brasil ha sido visto, como lo recalca Bratman (2015), como un jugador central en la política mundial ambiental. Sin embargo, debates internos lo han culpado a veces de frenar acuerdos ambientales mundiales. Por otro lado, como lo menciona Sachs (1996, p. 116) "Los países descubrieron que no eran unidades autocontenidas, sino que eran vulnerables a acciones tomadas por otros'. Con la creación del Tratado de la Cooperación Amazónica (TCA), en 1978, Brasil exhibió gran proactividad en liderar un proceso que buscaba mostrar al Norte que los Estados amazónicos podrían hacerse cargo de sus asuntos. La concepción de un "desarrollo armónico" e "integral" (TCA, 1978) en un marco común regional prometía hacer de la Amazonía un espacio de cooperación menos asimétrico. Posteriormente, 
la Organización del TCA buscó, a finales de los años noventa, solidificar la búsqueda de consensos en la Amazonía. No hay que desconocer que las naciones amazónicas han actuado juntas, buscando posiciones comunes en foros internacionales (Abdala, 2007). Sin embargo, la Amazonía sigue siendo un espacio vulnerado y vulnerable, manteniendo sobre ello, todavía en el siglo XXI, el "fantasma de la internacionalización".

\section{Conclusiones}

Vimos que el fantasma de la internacionalización de la Amazonía, el cual en la práctica se plasmaría en la pérdida de la soberanía absoluta de Brasil en ese espacio, se hizo sentir fuertemente en medios militares, académicos y políticos brasileños, durante la segunda mitad del siglo XX, llegando a las puertas del siglo XXI, cuando en la Estrategia Nacional de Defensa se menciona: "Quien cuida de la Amazonía brasileña, al servicio de la humanidad y de sí mismo, es Brasil." (Ministerio de Defesa Brasil, 2008, p. 14).La problemática de la preservación ambiental de la Amazonía plasma diferencias de enfoques entre el Norte y el Sur, las cuales muestran diferencias de capacidades, tecnología y conocimiento entre las grandes potencias y las naciones más débiles en el sistema internacional.

Brasil ha tenido un rol particular en los debates internacionales sobre Medio Ambiente, pues ha liderado un movimiento de países del Sur que no están dispuestos a perder su soberanía en pro de lo que son considerados intereses del Norte en ricos recursos naturales. Sin embargo, no siempre la Amazonía brasileña ha sido protegida como debiera ser.

Ha sido fundamental en la importancia de que la Amazonía ha tomado en los debates internacionales el traslado de la discusión del medio ambiente de "la condición de res nullis (no pertenece a nadie) para la de res omnium (que pertenece a toda la sociedad)". La problemática al respecto radica también en las discusiones alrededor de que los países en vías de desarrollo piden condiciones en igualdad de circunstancias, como el caso de insertar en las mismas negociaciones las selvas tropicales y los bosques temperados.

En Brasil, las mencionadas discrepancias de poder en el sistema internacional fueron, quizás más que en cualquier otra nación sudamericana, visualizadas como una amenaza a su soberanía, sobre todo por parte de militares, políticos y académicos.

Varios autores brasileños llamaron la atención por el hecho de que las naciones más poderosas ampliaron sus intereses y sus estrategias de seguridad en el ámbito externo, traspasando fronteras nacionales, lo cual afectaría la soberanía de los países en territorios de selvas tropicales.

Como resultado de esta disparidad de poder en el sistema mundial, el debate 
sobre la protección de selvas tropicales sigue siendo un escollo para el buen entendimiento entre el Norte y el Sur. De igual forma esta investigación demostró que académicos de universidades de naciones del Norte tienen mayor propensión para proponer una restricción efectiva, por medio de un fideicomiso de Estados, en cuanto a la soberanía permanente sobre los recursos, la cual ya está plasmada en la Convención del Patrimonio Mundial.

En este caso, tal como la Antártica, las selvas tropicales estarían bajo un fideicomiso de varias naciones. La solución para proteger recursos de preocupación común (common concern) conllevaría, muy probablemente, a una reconfiguración de la soberanía territorial. Estas propuestas, sin embargo, no serían de agrado de Estados como Brasil.

Finalmente, es importante mencionar que por medio de la revisión de fuentes primarias de organizaciones internacionales y documentos oficiales brasileños fue posible verificar las discrepancias de posiciones y enfoques entre países desarrollados y naciones en vías de desarrollo. Esto no es necesariamente negativo pero plasma que estas divergencias no pudieron ser, hasta la fecha, superadas en pro de la protección de la Amazonía.

Brasil tiene un papel clave como puente entre el Sur y el Norte. La cuestión es si sabrá aprovechar su especial posición en el ámbito internacional para trabajar en pro de la preservación de la Amazonía.

Es necesario seguir realizando investigaciones para definir y analizar cuáles aspectos están obstaculizando el diálogo entreel Suryel Norteentemáticas ambientales, tan fundamentales para la Amazonía brasileña.

\section{Referencias}

Abdala, F. (2007), Governança Global sobre as Florestas: O Caso do Programa Piloto para a proteção das Florestas Tropicais do Brasil-PPG7, Tesis de Doctorado, Universidad de Brasilia.

Agência do Senado, 16 de julio de 2015, ponencia del general Eduardo Villas Boas sobre la soberanía de Brasil en la Amazonía. Recuperado en maio de 2016. Disponible en:

http://www12.senado.leg.br/ noticias/materias/2015/07/16/ para-comandante-do-exercitosoberania-sobre-a-amazoniaenfrenta-deficits

Altinbas, D. (2013), South-South Cooperation: a Counterhegemonic Movement?, en Dargin, J. (ed.), Rise of the Global South: Philosophical, Geopolitical and Economic Trends of the $21^{\text {st }}$ Century, Singapura: SG, 29-66.

Amorim, C.; Rezende, S., Silva, M., "A Amazônia não está a venda", 
Folha de São Paulo, 17.10.2006. Recuperado en abril de 2016. Disponible en: http://www1. folha.uol.com.br/fsp/opiniao/ fz1710200608.htmn

Anatol. A. (2011), Die Organisation der brasilianischen Grensicherheitpolitik, Wiesbaden: VS Verlag.

Ayoob, M. (2002), "Inequality and Theorizing in International Relations: The Case for Subaltern Realism", en International Studies Review, 4, 3, 27-48.

Becker, B. (2004), Amazônia.

Geopolítica na Virada do III Milénio, Garamond: Rio de Janeiro.

Bosselmann, K. (2016), The Principle of Sustainability: Transforming Law and Governance, Londres: Routledge.

Bratman, E. (2015), "Brazil' s Ambivalent Challenge to Global Environment Norms", en Stuenkel, O. y Taylor, M. (eds), Brazil on Global Stage, New York: Palgrave MacMillan, 95-116.

"Brazil to Call for Global Fund to Save Rainforests and Cut Climate Change", The Guardian, 19/10/2006. Recuperado en abril de 2016. Disponible en: https://www.theguardian.com/ environment/2006/oct/19/brazil. onservationandendangeredspecies

Cárdenas, F.A. y Cadena, F. (2009), "Desafíos impuestos por el Derecho Internacional Ambiental al Derecho Internacional Clásico", en ACDI, 2, 141-174.
Castro, E. (2008), "A Amazônia e seu Lugar Central na Integração Sul-Americana" en, Nascimento, D. (ed.), Relações Internacionais e Defesa na Amazônia, Belém: NAEA/UFPA, 21-46.

Conca, K. (1995), "Environmental Protection, International Norms and State Sovereignty: The Case of the Brazilian Amazon", en Lyons, G. y Mastanduno, M. (ed.), Beyond Westphalia? State Sovereignty and International Intervention, Baltimore: John Hopkins Univ. Press, 147-169.

Convenio sobre Diversidad Biológica, Naciones Unidas, 1992. Recuperado el 10 de marzo de 2016. Disponible en:

https://www.cbd.int/doc/legal/cbd-es. pdf

Correa do Lago, A. (2006), O Brasil e as três Conferências Ambientais das Nações, Unidas, Brasilia: Instituto Rio Branco/FUNAG:

Costa-Filho, A. (2003), "Uma Nova "OTCA" sob Velha Ótica", en Aragón, L.E. y Clüsener-Godt, M. (eds), Problemática do Uso Local e Global da Água da Amazônia, Belém: NAEA/UNESCO, 383-396.

Da Silva, R.I. (2012), A Cooperação Multilateral entre os Países Amazônicos: Atuação do Tratado de Cooperação Amazônica, Tesis de Maestría, São Paulo: Universidade Estadual Paulista Júlio de Mesquita Filho. 
Da Silva Guevara, G. (2011), "Brasil, opciones estratégicas de una potencia emergente para afirmar su liderazgo Mundial", Oasis, 16, 5-23.

Declaración de Estocolmo sobre el Medio Ambiente Humano, 16 de junio de 1972. Recuperado en febrero de 2016. Disponible en: http://www.ordenjuridico.gob.mx/ TratInt/Derechos\%20Humanos/ INST\%2005.pdf

Declaración de Rio (1992) sobre el Medio Ambiente y Desarrollo. Recuperado en febrero de 2016. Disponible en: http://www.un.org/ spanish/esa/sustdev/agenda21/ riodeclaration.htm

Duarte, A.P. (2014), A "Estrategia NacionaldeDefesa" eo Pensamento Estratégico Contemporâneo do Brasil, en Barroso, L. y Escorrega, L. (eds), Estudos de Homenagem a Abel Cabral Couto, Lisboa: IESM, 53-77.

Eckersley, R. (2009), "Ecological Intervention: Prospects and Limits", en Rosenthal, J. y Barry, C. (eds), Ethics and International Affairs, Washington D.C.: Georgetown Univ. Press, 131-152.

Estrategia Nacional de Defesa, Brasil, 2008. Recuperado en marzo de 2016. Disponible en Ministerio de Defensa de Brasil:http:// www.defesa.gov.br/projetosweb/ estrategia/arquivos/estrategia_ defesa_nacional_portugues.pdf
Estrategia Nacional de Defesa, 2012. Recuperado en marzo de 2016. Disponible en: http://www.defesa. gov.br/arquivos/2012/mes07/end. pdf

Exército Brasileiro, Comando Militar do Norte: Operação Cabo Orange, 30 de maio a 10 de junio de 2016. Recuperado el 5 de julio de 2016. Disponible en: http://www.eb.mil. br/web/resiscomsex/cmn/-/asset publisher/PyGpfR13fXLW/content/ comando-militar-do-norteoperacao-cabo-oran-1 ? redirect $=$ http $\% 3$ A \% 2F\% 2 Fwww.eb. mil.br\%2 Fweb\%2 Fresisco msex\%2Fcmn\%3Fp_p_d\%3D101. INSTANCE_PyGpfR13fXLW\%26p _p_lifecycle\%3D0\%26p_p_state $\%$ 3Dnormal\%2

Foresta, R. (1992), "Amazonia and the Politics of Geopolitics", Geographical Review, 82, 2, 128142.

García, B. (2011), The Amazon from an International Law Perspective, New York: Cambridge University Press.

Herrera Montañez, L. C. (2011), Implicaciones de la Amazonía en la Política Exterior de Brasil, Tesis de Maestría, Universidad Javeriana.

Informe Brundtland, Naciones Unidas, 1987, disponible en: http://www. un-documents.net/ocf-02.htm\# I

Informe del Consejo Económico y Social del Foro de las Naciones Unidas sobre los Bosques, junio 2008. 
-Informe del Instituto de Pesquisa Ambiental (IPAM), Brasilia, 11 de abril de 2016.

"Internationalization of the world: Cristovam Buarque", 7 de maio de 2008. Recuperado en maio de 2016. Disponible en: https://1drop. wordpress.com/2008/05/07/ internationalization-of-the-worldcristovam-buarque/

Kiss, A. y Shelton, D. (2007), Guide to International Environmental Law. Leiden: Martinus Nijhoff Pub.

Krasner, S. (1999), Sovereignty, Organized Hypocrasy, Princeton: Princeton Univ. Press.

Liftin, K. (1997), "Sovereignty in World Ecopolitics", en Mershon International Studies Review, 41, 167-204.

Lima de Medeiros, R.A. (2012), Decodificando a internacionalização da Amazônia em Narrativas e Práticas Institucionais, Tesis Doctorado, Universidad de Brasilia.

Madeira, W. (2014), "Plano Amazônia Sustentável e Desenvolvimento Desigual", en Ambiente \& Sociedade, XVII, no. 3, Jul.-Set., 19-34.

Meira Mattos, A. (1995), Da Amazônia Brasileira a Outros Estudos, Belém: Cejup. (2008), Direito das Organizações Internacionais e Direito de Integração, Rio de Janeiro: Renovar.
Mercado-Jarrín, E. (1978), "Pacto Amazónico ¿Dominación o Integración?", en Nueva Sociedad, 37, 5.18 .

Ministério do Meio Ambiente, Deforestación en la Amazonia legal. Recuperado en abril de 2016. Disponible en: http://www. mma.gov.br/mma-em-numeros/ desmatamento

Mische, P. (1989), "Ecological Security and the need to reconceptualize sovereignty", Alternatives, 4, 389427.

Miyamoto, S. (2008), Amazônia, Política e Defesa en, Nascimento, D. (ed.), Relações Internacionais e Defesa na Amazônia, Belém: NAEA/UFPA, 65-98.

Ovando. N. (2009), "La Selva Amazónica debe ser Patrimonio de la Humanidad", BWN Patagonia, 27/06, disponible en http:// bolsonweb.net/diariobolson/ detalle.php?id_noticia $=22360$

Plano Desenvolvimento Sustentável (PAS) disponible en: http:// www.mma.gov.br/estruturas/ sca/_arquivos/plano_amazonia_ sustentavel.pdfMinisterio do Meio Ambiente, recuperado el 10 de agosto.

Passos, P. (2009), "A Conferencia de Estocolmo como Ponto de Partida para a Proteção Internacional do Meio Ambiente", en Revista Direitos Fundamentais e Democracia, 6, 1-25. 
Plan Calha Norte, Ministerio da Defesa. Recuperado en junio de 2016. Disponible: http://www. defesa.gov.br/programas-sociais/ programa-calha-norte

Pueblos indígenas de la Amazonía, recuperado el 8 de agosto de 2016. Disponible en: http://otca.paginaoficial.com/amazon/our_amazon

Rabinovici, A. (2011), Militantes e Militares na Midia: Questões sobre a Soberania da Amazônia Brasileira, en Teoria e Pesquisa, 20, 1, 99-116.

Report of the International Commission on Intervention and State Sovereignty (2001). The Responsibility to Protect, Otawa: International Development Research Center.

Recuperado en febrero de 2016. Disponible en: http://responsibility toprotect.org/ICISS\%20Report.pdf

Ricupero, B. i.a. (1995), Meio Ambiente e Dívida Externa: Anotações de uma Pesquisa, Cadernos CEDEC, 40.

Rocha Paiva, L.E. (2012), "Perspectivas de Conflitos na Amazônia e Reflexos para a Defesa Nacional", en Seminario de Segurança da Amazônia, Brasilia: Secretaria de Assuntos Estrategicos, 31-51.

Rothwell, D. (1990), "The Antartic Treaty System: Resource Development, Environmental Protection or Disintegration?", en Artic, 43, 3, 284-291.
Ryan, M. (1993), Calha Norte: Explaining Brazilian Army Presence in the Amazon, Tesis de Maestría, Monterrey: Naval Postgraduate School.

Sachs, W. (1996), "Medio ambiente", en Sachs, W. (ed.), Diccionario del Desarrollo, Perú: PRATEC, 115132.

Schiffman, R. (2015), La Deforestación en la Amazonía se incrementa, Scientific American, 30.06.2015. Recuperado en abril de 2016. Disponible en:

http://www.scientificamerican.com/ espanol/noticias/la-deforestacionen-la-amazonia-se-incrementa/

Schreurs, M. (1997), "Conservation Development, and State Sovereignty", en Hashmi, State Sovereignty, Change and Persistence, Pennsilvania: Penn Uni Press, pp. 182-204.

Schrijver, N. (2009), "After us the Deluge? The Position of Future Generations of Humankind in International Environment", en Salih, M. (ed.), Climate Change and Sustainable Development, Cheltenham: Edward Elgar, pp. 59-78.

Secreto, M. V. (2007), A Ocupação dos "Espaços Vazios" no Governo Vargas: do "discurso do Rio Amazonas" à saga dos Soldados da borracha, en: Estudos Históricos, 40, 115-135.

Stec, S. (2010), Humanitarian Limits to Sovereignty: Common 
Concern and Common Heritage. Approaches to Natural resources and Environment, International Community Law Review, 12, 361389.

Teixeira da Silva, F. C. (2008), A Amazônia e as Novas Ameaças Mundiais, en Nascimento, D. (ed.), Relações Internacionais e Defesa na Amazônia, Belém: NAEA/UFPA, 47-64.

Villas Boas, E. (2013), Meira Mattos, a Amazônia e o Livro, en Cadernos Estratégicos, 12, 43-75.

"Whose Rain Forest is this, Anyway?", New York Times, 18/05/2008.

Recuperado en mayo de 2016. Disponible en: http://www.nyti mes.com/2008/05/18/weekin review/18barrionuevo.html?_r=0 